\title{
Neutral Hydrogen in HCG 95
}

\author{
W. K. Huchtmeier
}

Max-Planck-Institut für Radioastronomie, Bonn, D-53121, Germany

Lourdes Verdes-Montenegro

Instituto de Astrofísica de Andalucía, Granada, Spain

Min Yun

National Radio Astronomy Observatory, Socorro, N.M., U.S.A.

A. del Olmo and J. Perea

Instituto de Astrofísica de Andalucía, Granada, Spain

\begin{abstract}
.
We have observed $\mathrm{H}$ I emission and radio continuum emission from the compact group of galaxies HCG 95 with the Very Large Array (VLA) ${ }^{1}$. Two continuum sources coincide in with galaxies in this group: HCG $95 \mathrm{~B}$ $(3.9 \mathrm{mJy})$ and HCG $95 \mathrm{C}(6 \mathrm{mJy})$. H I emission and absorption was detected in galaxy HCG $95 \mathrm{C}$. In addition we detected two so far unknown dwarf galaxies by their $\mathrm{H}$ I emission within 3.5 arcmin of the group center. We did not detect galaxy $\mathrm{b}$ (with $v=8000 \mathrm{~km} \mathrm{~s}^{-1}$ it is obviously a foreground object) and galaxy $d$ - an edge-on Sc galaxy. This group definitely is $\mathrm{HI}$ deficient compared with the average $\mathrm{HI}$ content expected for spiral galaxies of the same luminosity and type. The first-ranked elliptical galaxy HCG $95 \mathrm{~A}$ might be responsible for the observed $\mathrm{H}$ I deficiency in this group.
\end{abstract}

\section{Introduction}

The galaxy density in compact groups is similar to that in central areas of galaxy clusters. Over $70 \%$ of the galaxies in Hickson Compact Groups (HCG, Hickson 1982) are spirals and irregulars (Rood and Stroble 1994). Hence, there is a high probability for interaction and mergers with large amounts of interstellar matter involved. Compact groups are an ideal laboratory to investigate the effects of interaction and mergers between two or three galaxies. Roughly $7 \%$ of the galaxies in HCG's are in the process of merging (Zepf 1993). A few

\footnotetext{
${ }^{1}$ The National Radio Astronomy Observatory is operated by Associated Universities, Inc., under contract with the National Science Foundation.
} 


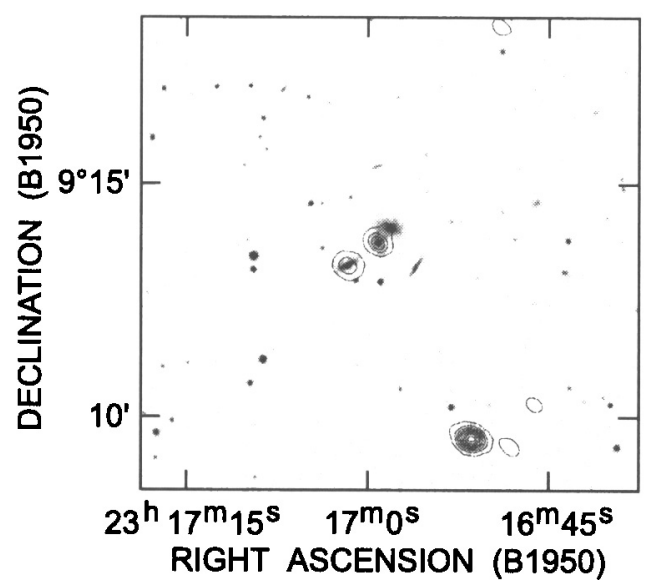

Figure 1. Radio continuum sources at 21-cm wavelength in the area of HCG 95 are shown as contours on a Digital Sky Survey print. Two continuum sources in the center correspond to $\mathrm{H} 95 \mathrm{C} 1(6 \mathrm{mJy})$ and H95B (3.9 mJy).

definite mergers are HCG 18, 31, 95. HCG $95 \mathrm{C}$ has two nuclei (HCG $95 \mathrm{C} 1$ and HCG $95 \mathrm{C2}$ ) and shows four optical jets or bridges (e.g. Iglesias-Páramo and Vílchez 1998), a definite sign for a merger plus interaction as numerical simulations of interactions never produced more than two arms.

\section{Observations}

Observations were performed with the VLA in the CnB-array configuration, integrating for 4.5 hours yielded an r.m.s. noise of $0.35 \mathrm{mJy}$ per channel (channel separation of $22 \mathrm{~km} \mathrm{~s}^{-1}$ ) and a synthesized beam of $22 . " \times 16.2$ " (p.a. $49^{\circ}$ ). The 4IF observing mode was used with a bandwidth of $3.125 \mathrm{MHz}$ each shifted by 3 $\mathrm{MHz}$ relatively to each other and covering a velocity range from 11420 to 12535 $\mathrm{km} \mathrm{s}^{-1}$.

Table 1. H I parameters of the dwarf galaxies in HCG 95

\begin{tabular}{|lllcccc|}
\hline Name & R.A. (1950.0) Dec. & velocity & H I-flux & $\mathrm{S}_{\max }$ & line-width \\
\hline & & & $\mathrm{km} \mathrm{s}^{-1}$ & $\mathrm{Jy} \mathrm{km} \mathrm{s}^{-1}$ & $\mathrm{mJy}$ & $50 \%$ \\
\hline H95dwA & 231644.8 & 091415.5 & $11830 \pm 5$ & 0.33 & $2.4 \pm 0.35$ & 76 \\
H95dwB & 231657.2 & 091705.0 & $11730 \pm 5$ & 0.18 & $2.3 \pm 0.35$ & 55 \\
& & & & & & \\
\end{tabular}




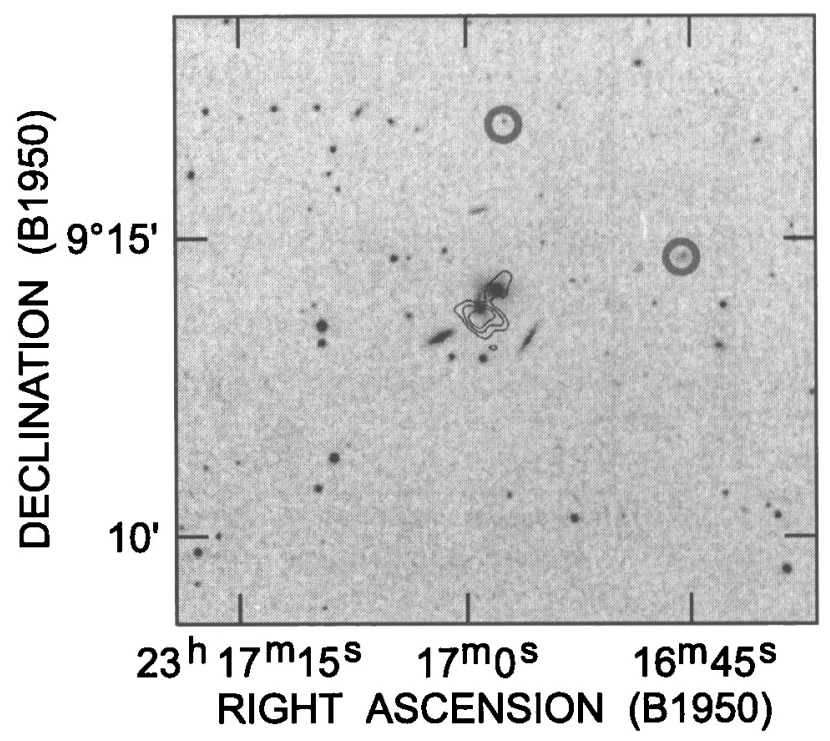

Figure 2. A reproduction from the DSS. Contours indicate where $\mathrm{H}$ I emission has been observed associated with HCG 95 C (in the radial velocity range 11420 to $12535 \mathrm{~km} \mathrm{~s}^{-1}$ ). Two dwarf galaxies (indicated by grey circles) about 3.5' from the center of the group have been observed for the first time.

\section{Data}

The radio continuum emission within a field 9' $\times 9$ ' around HCG 95 is presented in Fig. 1. The continuum was obtained by summing all channels free of line emission. Two radio continuum sources in the field center coincide in position with two galaxies of this compact group, the eastern source, HCG 95 B $(v=8000$ $\mathrm{km} \mathrm{s}^{-1}$; Iglesias-Páramo and Vílchez 1998, also confirmed by our own observations) has a flux of $3.9 \mathrm{mJy}$, the other source - HCG $95 \mathrm{C}$ (centered on $\mathrm{C} 1$ ) has a flux of $6 \mathrm{mJy}$.

$\mathrm{H}$ I emission has been detected in three galaxies: $\mathrm{HCG} 95 \mathrm{C}$, the merging galaxy $\mathrm{C}$ (with two nuclei $\mathrm{C} 1$ and $\mathrm{C} 2$ ) in gravitational interaction with the elliptical galaxy HCG 95 A and two dwarf galaxies so far unknown members of this group (Fig. 2).

Two spiral galaxies have not been detected in H I: galaxy HCG 95 B was missed as it was outside the observed bandwidth. Its new radial velocity of 8000 $\mathrm{km} \mathrm{s}^{-1}$ turns it into a foreground object. Galaxy HCG 95 D, an edge-on spiral (Sc), was not detected even after smoothing the data in frequency. Within the given noise up to 2 or $3 \times 10^{9} M_{\odot}$ of neutral hydrogen may be hidden depending on the actual rotational velocity of this galaxy. 
An obvious feature is the $\mathrm{HI}$ absorption against the central radio source (C1). From the absorption and the radio continuum flux of the source $\mathrm{C} 1$ we derive an optical depth of 0.287 which yields a column density of $4.6 \times 10^{21}$ $\mathrm{cm}^{-2}$ of $\mathrm{HI}$ assuming the canonical value of $100 \mathrm{~K}$ for the spin temperature. This column density is typical for disks of spiral galaxies.

\section{Discussion}

Ongoing active star formation is suggested by strong $\mathrm{H} \alpha$ emission in HCG $95 \mathrm{C} 1$, in the northern arm, and in the nucleus of the elliptical galaxy HCG 95 A (Iglesias-Páramo and Vílchez 1997). The presence of starbust in C1 is supported by the detection of associated radio continuum emission.

HCG 95 is H I-deficient as a group (Huchtmeier 1997), and these highresolution observations now allow the examination of gas content in each individual member. Based on their luminosity and Hubble type, the expected $\mathrm{HI}$ masses for the galaxy C \& D are about $10^{10} M_{\odot}$ and $8 \times 10^{9} M_{\odot}$, respectively (Huchtmeier \& Richter 1989). Only 3 to $5 \times 10^{9} M_{\odot}$ of $\mathrm{HI}$ is detected in galaxy $\mathrm{C}$, and only an upper limit of 2 to $3 \times 10^{9} M_{\odot}$ of $\mathrm{H} \mathrm{I}$ is derived for galaxy $\mathrm{D}$. Therefore the two spiral galaxies in HCG 95 are definitely $\mathrm{H}$ I-deficient by at least factors of 2 to 4 . Increased star formation, such as observed in galaxy HCG $95 \mathrm{C}$, may have depleted gas in this group. Alternatively, star formation may have increased the apparent luminosity of a galaxy, resulting in the over-estimate of the expected $\mathrm{M}_{H I}$. The compact group environment can affect the $\mathrm{H}$ I content of the group in a variety of other ways as well. For example, tidal capture of $\mathrm{H} \mathrm{I}$ from a passing neighbor or accretion of $\mathrm{H}$ l-rich dwarf galaxies might increase the relative $\mathrm{H}$ I-content of the group in some cases. Two such $\mathrm{H}$ I-rich neighbors are found in our search. The accretion of $\mathrm{H} \mathrm{I}$ by the first-ranked elliptical galaxy (HCG 95 A) and the subsequent consumption by star formation may have reduced the total $\mathrm{HI}$ content of the group (Verdes-Montenegro et al. 2000, this conference).

Acknowledgments. The NED database is supported at IPAC by NASA. The Digitized Sky Surveys were produced at the Space Telescope Science Institute under US Government Grant NAG W-2166.

\section{References}

Hickson, P. 1982, ApJ, 255, 382

Huchtmeier, W. K. 1997, A\&A, 325, 473

Huchtmeier, W. K., Richter, O.-G. 1989, A\&A, 210, 1

Iglesias-Páramo, J., Vílchez, J. M., 1997, ApJL, 489, L13

Iglesias-Páramo, J., Vílchez, J. M. 1998, AJ, 115, 1791, ApJS, 117, 1

Rood, H. J., Stroble, M. F. 1994, PASP, 106, 413

Verdes-Montenegro, Lourdes, Yun, M., Williams, B. A., Huchtmeier, W. K., del Olmo, A., Perea, J. 2000, this conference

Williams, B. A., Rood, H. J. 1987, ApJS, 63, 265

Zepf, S. E. 1993, ApJ, 407, 448 